\title{
Morfología funcional del fitoplancton en un lago de alta montaña tropical: Lago de Tota (Boyacá-Colombia)
}

Claudia Liliana Muñoz-López ${ }^{1}$, Nelson Javier Aranguren-Riaño ${ }^{1}$ \& Santiago R. Duque ${ }^{2}$

1. Grupo de Investigación Unidad de Ecología en Sistemas Acuáticos-UDESA, Escuela de Ciencias Biológicas, Universidad Pedagógica y Tecnológica de Colombia, Av. Central del Norte 39-115 AA 1094 Tunja, Colombia; clausmunoz92@gmail.com,nelson.aranguren@uptc.edu.co

2. Grupo de Investigación Limnología Amazónica, Instituto Amazónico de Investigaciones -Imani, Universidad Nacional de Colombia-Sede Amazonía, km 2 -Vía Tarapacá AA 215 Leticia, Colombia; srduquee@unal.edu.co

Recibido 26-IV-2016. Corregido 12-XII-2016. Aceptado 17-I-2017.

\begin{abstract}
Functional morphology of phytoplankton in a tropical high mountain lake: Tota Lake (BoyacáColombia). Functional morphology corresponds to adaptive responses to changes in the environment. In phytoplankton, traits such as the development of mucilage, oil vesicles, filaments and variation in the surface/volume ratio, allow algae to deal with sedimentation, optimizing light and nutrient uptake. The aim of this research was to evaluate the relationship between traits and variation in physical and chemical conditions in Tota Lake (Boyacá, Colombia). For this, ten samplings were undertaken in a monthly basis between October 2013 and July 2014. We measured water $\mathrm{pH}$, conductivity, temperature, dissolved oxygen, oxygen saturation, alkalinity and total hardness; we also quantified Total Kjeldahl nitrogen and Total phosphorus. Algae were collected at different depths according to Secchi transparency. Taxonomic composition and abundance were estimated by the chamber sedimentation method. Morphological traits were measured for each taxa according to geometric models, allowing traits classification into morphologically based functional groups (MBFG) and having algae biomass expressed as biovolume. The variables were evaluated through Principal Components Analysis, which included time-space effect and it was interpreted according to variation in water level. Through Canonical Correspondence Analysis we established the relationship between phytoplankton biovolume accumulated in form categories and environmental variables. The ordination showed that limnological conditions are influenced by seasonal changes, which are mainly represented by oxygen saturation percentage, Secchi transparency, TKN, hardness and alkalinity. Four MBFG were found: IV, V, VI and VII, being MBFG IV and VII the most representatives. ACC was significant (Monte-Carlo Test, $\mathrm{p}<0.05$ ). In conclusion, the morphological and functional response of phytoplankton in Tota Lake is driven by short-term variability in transparency, phosphorus, nitrogen, hardness, and electrical conductivity, which are all affected by seasonal changes in the lake level. Rev. Biol. Trop. 65 (2): 669-683. Epub 2017 June 01.
\end{abstract}

Key words: biovolume, functional group, functional morphology, phytoplankton, Tota Lake.

El fitoplancton es un conjunto de microorganismos, compuesto en su mayoría por especies fotoautotróficas, pertenecientes a varios linajes evolutivos y adaptadas a la suspensión en el agua. Es considerado la base de la cadena trófica y el principal receptor de energía en sistemas acuáticos (Harris, 1986; Graham, Graham, \& Wilcox, 2009; Reynolds, 2006; Esteves $\&$ Suzuki, 2011). Su distribución en la columna de agua está regulada principalmente por la incidencia de la luz, la temperatura, la disponibilidad de nutrientes, la competencia, la herbivoría y la dinámica hidráulica (Reynolds, 1980; Rhee, 1982; Margalef, 1983, Reynolds, 2006).

Para enfrentar las diferentes expresiones del hábitat en un ambiente, los organismos del fitoplancton poseen una serie de adaptaciones de forma o rasgos que se asocian a la denominada morfología funcional (Margalef, 1978; Brasil \& Huszar, 2011). Características 
morfológicas como: desarrollo de vaina mucilaginosa, vesículas de aceite (Esteves \& Suzuki, 2011), formación de filamentos y variación en la relación superficie/volumen $(\mathrm{S} / \mathrm{V})$, se incluyen en ésta y son importantes, pues permiten al fitoplancton enfrentar la sedimentación, optimizar la captación lumínica y de nutrientes (Reynolds, 1997). A su vez la morfología funcional, resulta en la asociación de organismos con rasgos similares o estrategias, que van a promover la supervivencia y el ajuste a ciertas presiones del medio (Salmaso \& Padisák, 2007; Roldán-Pérez \& Ramírez-Restrepo, 2008).

Desde el enfoque funcional, que se basa en la teoría de nicho ecológico (Hutchinson, 1957), se han desarrollado varias clasificaciones para el fitoplancton. Lewis (1976), reconoció la importancia ecológica de la relación (S/V) y la asoció con la captación de luz y nutrientes, como resultado de la selección natural y la competencia. Posteriormente, Margalef (1978) estableció que las diferentes "formas de vida" observadas en este grupo, son el factor determinante en la sucesión estacional del mismo e interpretadas como adaptaciones de supervivencia en un ambiente inestable y turbulento, relacionado a su vez, con el flujo de energía y el suministro de nutrimentos.

Reynolds (1980) evaluó el fitoplancton de seis ambientes ingleses: cuatro lagos naturales y dos reservorios experimentales. En este trabajo distinguió 14 "ensambles de especies", los cuales relacionó principalmente con la disponibilidad de nutrientes, la estabilidad de la columna de agua y las estrategias de vida. Además, concluyó que las especies de cada grupo o ensamble poseen atributos ecológicos que les permiten dominar o co-dominar en cada una de las etapas de la sucesión estacional. Posteriormente, adaptando el modelo de Grime (1977) para plantas terrestres, definió un nuevo sistema de clasificación funcional; categorizando a los organismos de este grupo biológico en estrategas $\mathrm{C}, \mathrm{R}$ y $\mathrm{S}$, estableciendo como rasgo distintivo la relación superficie/volumen (S/V) y la susceptibilidad al disturbio, al estrés y a la limitación de recursos (Reynolds, 1988).
Reynolds, Huszar, Kruk, Naselli-Flores y Melo (2002) actualizaron el sistema de clasificación funcional del fitoplancton, postulando 31 asociaciones: grupos de especies (en su mayoría polifiléticos) que responden de manera similar frente a cambios en el ambiente, debido en gran medida, a rasgos adaptativos compartidos. Para simplificar su uso, este modelo fue ajustado por Padisák, Crossetti y Naselli-Flores (2009), quienes propusieron nueve agrupaciones más e incluyeron descripciones más detalladas de los hábitats donde podrían ocurrir las especies.

El modelo de clasificación más reciente fue propuesto por Kruk et al. (2010) quienes relacionan VII Grupos Funcionales Basados en Morfología (GFBM o $M B F G$, por sus siglas en inglés). Éstos son determinados, siguiendo rasgos sencillos como volumen, superficie, dimensión linear máxima y presencia de estructuras como: vainas mucilaginosas, flagelos, vesículas de gas (aerótopos), heterocistos y estructuras exo-esqueléticas de sílice. Estos atributos, guardan relación ecológica, más que filogenética; aspecto clave que permite interpretar los procesos que se dan en sistemas acuáticos (Weithoff, 2003; Kruk et al., 2010).

Recientemente, la ecología del fitoplancton de sistemas tropicales ha incorporado el fundamento de los modelos de clasificación funcional. Algunos estudios realizados en este tipo de ambientes, han sido desarrollados en el lago de inundación brasilero 'Batata', los cuales han concluido que las propiedades físicas del medio, son altamente determinantes sobre las estrategias o asociaciones del grupo en mención (Huszar \& Reynolds, 1997; Huszar, Silva, Marinho, Domingos, \& Sant'Anna, 2000; Melo \& Huszar, 2000). En el lago profundo natural 'Dom Helvécio', Souza, Barros, Barbosa, Hajnal y Padisák (2008) sugieren que el fitoplancton allí presente, está condicionado por cambios estacionales de la temperatura del agua, que promueven la mezcla e incrementan la disponibilidad de nutrientes. Proponen además, que el buen entendimiento de la relación ensamble fitoplanctónico-cambios medioambientales-períodos climáticos, puede 
ser una buena herramienta para el manejo de la calidad del agua, cuando se evalúan ecosistemas acuáticos del trópico.

Para el Lago de Tota, el registro más aproximado, menciona que en cuerpos de agua situados en la alta montaña, profundos y de baja temperatura, se establecen algas grandes y sin rasgos funcionales particulares, vinculadas en su mayoría, a los GFBM IV y VII (Hernández-Atilano et al., 2012).

Este lago de la alta montaña andina, es un ecosistema estratégico por la variedad de servicios que ofrece. Soporta una serie de tensores ambientales, relacionados principalmente con la ampliación de las fronteras disponibles para la agricultura, descarga de productos agroquímicos y de aguas residuales (Cordero, Ruiz, \& Vargas, 2005; Noriega, Castillo, Vásquez, \& Monroy, 2010), fenómenos que pueden modificar las condiciones naturales de los sistemas acuáticos (Smith \& Schindler, 2009), siendo el fitoplancton sensible a estos cambios, dadas sus variadas respuestas de adaptación (Duque \& Donato-Rondón, 1992). Según Brasil y Huszar (2011), el abordaje de los rasgos funcionales de las algas y su relación con su ambiente, permitiría establecer patrones o "predicciones" de la dinámica de los sistemas acuáticos.

Partiendo de este principio, se planteó como objetivo evaluar aspectos de la morfología funcional del fitoplancton y su relación con la variación de las condiciones físicas y químicas del lago de Tota, como una aproximación al entendimiento de su funcionamiento.

\section{MATERIALES Y MÉTODOS}

Área de estudio: El lago de Tota está ubicado sobre la cordillera Oriental, hacia el suroriente del departamento de Boyacá-Colombia (5³3'0” N - 72॰55'0” W), a 3015 msnm, entre la jurisdicción de los municipios de Aquitania, Cuítiva y Tota. Es un sistema de origen tectónico (Rangel \& Aguirre, 1983) con influencia glaciar, presenta una profundidad media de $30 \mathrm{~m}$ y máxima de $61 \mathrm{~m}$, un perímetro de 47 $\mathrm{km}$, un espejo de agua de 6000 ha, un volumen máximo de 1920 x $10^{6} \mathrm{~m}^{3}$ y se divide en dos cubetas interconectadas: lago Chico y lago Grande (Armada Nacional, 1998). La cuenca abarca una superficie de $201 \mathrm{~km}^{2}$ y ocupa una gran depresión en alta montaña (Cañón \& Valdés, 2011).

Es clasificado como un sistema polimíctico cálido y con tendencia a aumentar su estado trófico (Cardozo, Bermúdez, Aranguren-Riaño, \& Duque, 2005). El patrón de lluvias y de evaporación es monomodal: los meses de altas precipitaciones se registran entre abril y noviembre; los meses de baja precipitación de diciembre a marzo. La humedad relativa promedio multianual es de $82 \%$ (Cordero et al., 2005; Hernández-Atilano et al., 2013).

\section{Diseño de muestreo y medición de varia-} bles físicas y químicas: Se realizaron diez muestreos con periodicidad mensual, abarcando varios momentos de la cota del nivel (Fig. 1), entre octubre 2013 y julio 2014, en dos estaciones de la zona limnética del lago de Tota: una en lago Grande y otra en lago Chico. En cada una, se midió la transparencia Secchi y en la capa superficial la alcalinidad y la dureza (kits Aqua-Merck), el pH (pH-metro Schott D 55014), la conductividad eléctrica (conductímetro YSI 85) y la temperatura, oxígeno disuelto y porcentaje de saturación empleando un oxímetro YSI 55 (Wetzel \& Likens, 2000; Ruiz, 2002).

Para la cuantificación de nitrógeno total Kjeldahl (NTK) y fósforo total (PT), se tomaron $500 \mathrm{~mL}$ de agua integradas de tres profundidades: superficie, una profundidad Secchi (1DS) y tres profundidades Secchi (3DS). Se almacenaron en frascos de vidrio color ámbar, preservaron con ácido sulfúrico hasta obtener un $\mathrm{pH}$ cercano a 2 y se mantuvieron refrigeradas hasta su análisis (Wetzel \& Likens, 2000). Los métodos de cuantificación empleados en laboratorio fueron: Macro-Kjeldahl SM 4500Norg C, 4500-NH3 B, F, para NTK y Digestión Colorimétrica del ácido ascórbico SM 4500-P B, E, para PT (APHA, AWWA, \& WEF, 2012).

Muestreo del fitoplancton: Las muestras cuantitativas fueron obtenidas con botella tipo 


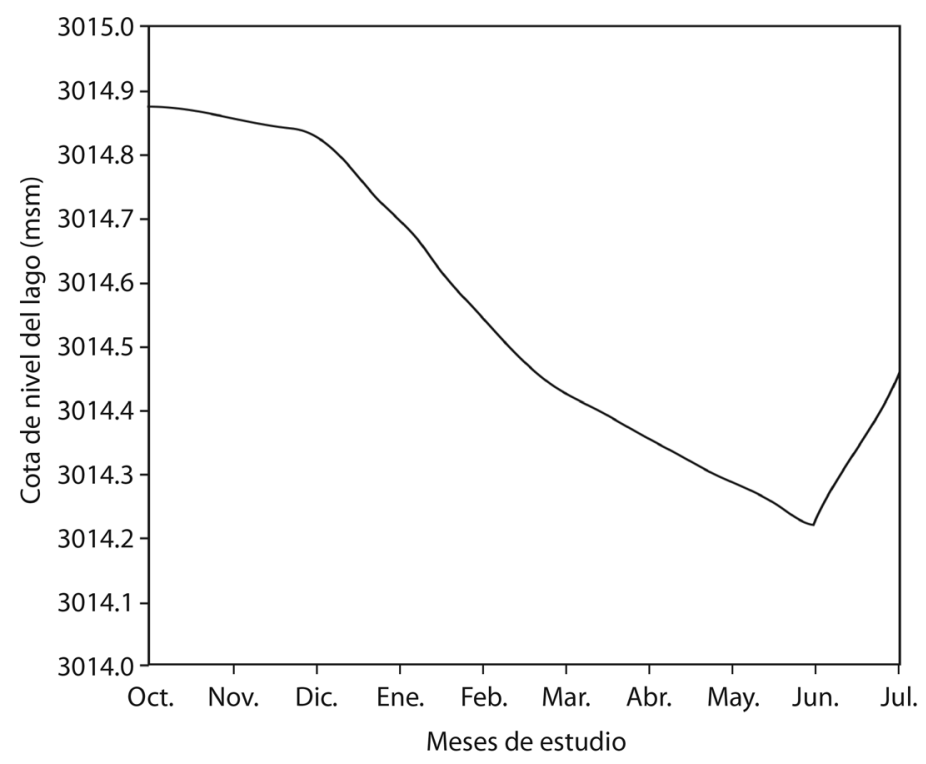

Fig. 1. Variación de la cota de nivel del lago para los meses de estudio (Cañón, com. pers.).

Fig. 1. Variation of Lake level elevation for the months of study (Cañón, com. pers.).

Schindler, en las profundidades definidas para nutrientes. Se colectó un volumen de $200 \mathrm{~mL}$, se almacenó en frascos ámbar y se preservó con lugol, en proporción de $1 \mathrm{~mL}$ por cada $100 \mathrm{~mL}$ de muestra (Schwoerbel, 1975). Además, se colectaron muestras cualitativas concentradas mediante arrastres superficiales con red de $17 \mu \mathrm{m}$ de diámetro de poro. Estas fueron fijadas con solución Transeau, en proporción 1:1 (Ramírez-Restrepo, 2000).

Procesamiento de muestras de fitoplancton: Se identificó el fitoplancton hasta género y/o especie, mediante el uso de claves especializadas y observaciones en un microscopio óptico (Nikon Eclipse E200) provisto de ocular micrométrico, a una magnificación de 400X. Para la evaluación cuantitativa, se implementó el método de sedimentación en cámaras de volumen conocido, para el caso 20 $\mathrm{mL}$ de muestra y observación en el microscopio invertido Olympus CKX41, (Utermöhl, 1958). Se contaron campos al azar, con una magnificación de 200X, con un mínimo de cuatrocientos individuos del morfotipo más frecuente (Ramírez-Restrepo, 2000). Cada filamento, cenobio o colonia se contó como individuo según Gómez, Rivera, Duque, Andrade y Rodríguez (2011). La densidad se expresó en términos de organismos por mililitro (org. $\mathrm{mL}^{-1}$ ), mediante la ecuación de Ros (1979):

$$
\text { org. } \mathrm{mL}^{-1}=\text { n.F/s.c.h }
$$

Donde:

$\mathrm{n}=$ número de organismos contados

$\mathrm{F}=$ factor de conversión $\left(10^{3} \mathrm{~mm} / \mathrm{mL}\right)$

$\mathrm{s}=$ superficie del campo óptico $\left(\mathrm{mm}^{2}\right)$

$\mathrm{c}=$ número de campos contados

$\mathrm{h}=$ altura de la cámara $(\mathrm{mm})$

Determinación de rasgos morfológicos, grupos funcionales y biovolumen: Se estimó la superficie (S), el volumen (V) y la dimensión linear máxima (DLM) de 49 taxa de fitoplancton, a partir de las medidas realizadas a treinta células (entre una y cinco para taxa poco comunes), según la correspondencia a los modelos geométricos y su respectiva representación algorítmica (Hillebrand, Dürselen, Kirschtel, Pollingher, \& Zohary, 1999; Sun \& Liu, 2003). La relación superficie/volumen $(\mathrm{S} / \mathrm{V})$ se calculó a partir del cociente de estas magnitudes. 
CUADRO 1

Categorías de forma modificadas de Alba y Jiménez (com. pers.)

TABLE 1

Form categories modified from Alba y Jiménez (com. pers.)

\begin{tabular}{cll} 
Categoría & \multicolumn{1}{c}{ Descripción } & \multicolumn{1}{c}{ Ejemplo } \\
A & Esfera & Asterococcus sp., Sphaerocystis sp. \\
B1 & Cilíndro + Elipsoide + Conos & Ceratium hirundinella \\
B2 & Media esfera & Staurodesmus connatus, Staurodesmus dejectus \\
C & Esfera prolongada & Oocystis cf. parva, Scenedesmus ecornis \\
D & Cilíndro & Aulacoseira granulata, Mougeotia sp. \\
E & Doble cono & Closterium acutum, Elakatothrix cf. gelatinosa, Monoraphidium cf. minutum \\
E1 & Doble cono truncado & Staurastrum chaetoceras, Staurastrum cingulum \\
F & Caja rectangular & Asterionella formosa, Tabellaria sp. \\
H & Prisma sobre paralelogramo & Nitzchia sp1. \\
\hline
\end{tabular}

Los taxa fueron clasificados en Grupos Funcionales Basados en Morfología (GFBM), siguiendo la clave dicotómica propuesta por Kruk et al. (2010). Se estimó la biomasa de las algas expresada como biovolumen a partir del producto del volumen geométrico calculado y la densidad de individuos del recuento (Rott, 1981; Kruk et al., 2010). A partir de esto, se evaluó el aporte de biovolumen por cada GFBM y por cada categoría de forma (Cuadro 1).

Tratamiento de la información: Para establecer el comportamiento de las variables físicas y químicas, se realizó un Análisis de Componentes Principales (ACP) (McCune \& Grace, 2002), que incluyó el efecto espaciotemporal y se interpretó en función de las variaciones del nivel del lago.

Mediante Análisis de Correspondencia Canónica (ACC), herramienta multivariada que facilita identificar la relación entre las variables biológicas y ambientales (Ter Braak, 1986; McCune \& Grace, 2002), se evaluó la dependencia del biovolumen obtenido, agrupado en categorías de forma (Cuadro 1) y la expresión física y química de cada estación y período de estudio. La significancia estadística del modelo se efectuó mediante el test MonteCarlo con 999 permutaciones (Castellanos \& Donato-Rondón, 2008). Previamente, la matriz biológica fue normalizada mediante la función Log x (García-Berthou, Alcaraz, Benejam, \& Benito, 2009). Los datos se analizaron en el software R (versión libre).

\section{RESULTADOS}

Variables físicas y químicas: $\mathrm{El} \mathrm{ACP}$ explicó el $53.07 \%$ de la varianza en los dos primeros componentes: $36.15 \%$ y $16.92 \%$, respectivamente y permitió establecer asociaciones entre variables, épocas de estudio y sitios de muestreo. El componente uno se asoció positivamente con el porcentaje de saturación del oxígeno disuelto $(\mathrm{r}=0.535)$, el oxígeno disuelto $(\mathrm{r}=0.514)$ y negativamente con la transparencia Secchi $(\mathrm{r}=0.223)$. El componente dos, con el nitrógeno total Kjeldahl $(\mathrm{r}=0.526)$, el $\mathrm{pH}(\mathrm{r}=0.357)$ y negativamente con la dureza $(\mathrm{r}=0.548)$ y la alcalinidad $(\mathrm{r}=0.434)$.

El plano de ordenación mostró la formación de cuatro grupos correspondientes con la variación temporal así: el primero agrupó a los muestreos de octubre y noviembre que se caracterizaron por menor transparencia Secchi, valores bajos de fósforo total y alta temperatura; éstos correspondieron al momento de alta cota de nivel. El segundo relacionó a los muestreos de febrero, marzo, abril y mayo cuya característica común conductividad eléctrica, 
$\mathrm{pH}$, oxígeno disuelto y porcentaje de saturación altos. Además, de coincidir con la reducción de la cota de nivel.

El tercer momento integró a los meses de junio y julio. Para éstos, la cota de nivel registró su nivel más bajo (3 $014.22 \mathrm{msnm}$ ) y un leve ascenso, respectivamente, y fueron explicados por valores altos de la transparencia Secchi y NTK. El cuarto, a diciembre y enero que se definieron por un descenso de nivel, temperatura y conductividad eléctrica bajas (Fig. 2).

\section{Rasgos morfológicos, grupos funciona-} les y biovolumen: En la figura 3 se representa la relación de la DLM y la S/V de los 49 taxa medidos, separadas por grupo funcional. La mayoría de éstos, presentaron baja relación superficie/volumen $\left(<1 \mu \mathrm{m}^{-1}\right)$. Sin embargo, en Cosmarium contractum, Closterium acutum, Closterium sp., Monoraphidium cf. contornum, Monoraphidium cf. minutum y Staurastrum leptocladum vinculadas al GFBM IV, este cociente fue mayor a $1 \mu \mathrm{m}^{-1}$. Este patrón también se observó en Cryptomonas cf. ovata, Gymnodinium sp. (GFBM V), Tabellaria sp., y Asterionella formosa (GFBM VI). Las dimensiones lineares máximas más altas (superiores a $170 \mu \mathrm{m}$ ), correspondieron a algas similares a un cilindro: Mougeotia sp., Phormidium sp., Aulacoseira granulata y Ceratium hirundinella y a doble cono truncado (C. acutum).

Se identificaron cuatro Grupos Funcionales Basados en Morfología (Fig. 4). Los más representativos fueron los GFBM IV y VII, los cuales fueron constantes temporalmente tanto en lago Grande como en lago Chico. Aunque hay variaciones sutiles, como ocurre en los meses de octubre y noviembre, donde la cota de nivel presenta sus valores máximos (3 $014.88 \mathrm{msnm}$ ), el grupo VII se acentuó más, sobretodo en el lago Chico. Al reducirse la cota, se evidenció un recambio por el

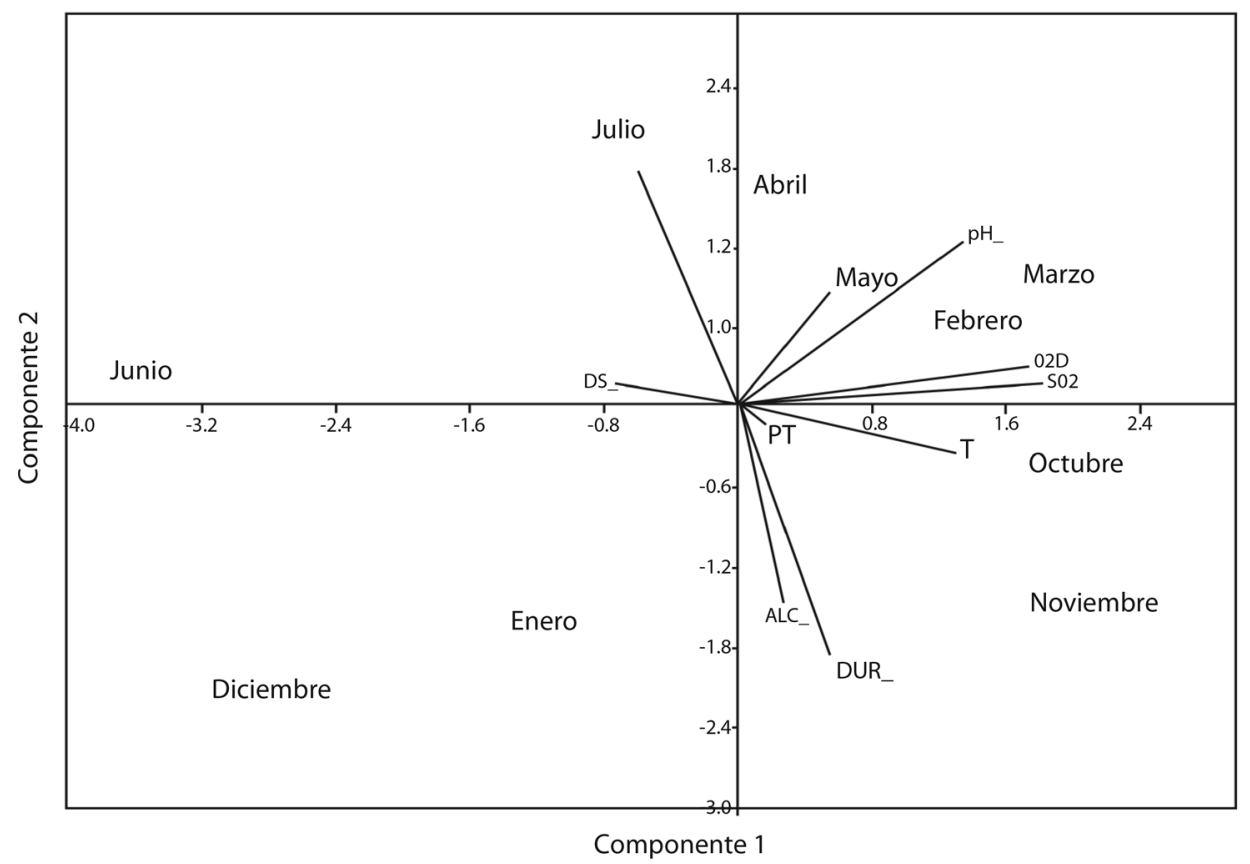

Fig. 2. Análisis de Componentes Principales (ACP) para las variables físicas y químicas obtenidas en los sitios de estudio. DS: transparencia Secchi; ALC: alcalinidad; DUR: dureza; C-E: conductividad eléctrica; T: temperatura; O2D: oxígeno disuelto; SO2: saturación de oxígeno; PT: fósforo total; NTK: nitrógeno total Kjeldahl.

Fig. 2. Principal Component Analysis (PCA) for the physical and chemical variables obtained in the study sites. DS: Secchi transparency; ALC: alkalinity; DUR: hardness; C-E: electrical conductivity; T: temperature; O2D: disolved oxygen; SO2: oxygen saturation; PT: total phosphorus; NTK: total Kjeldahl nitrogen. 


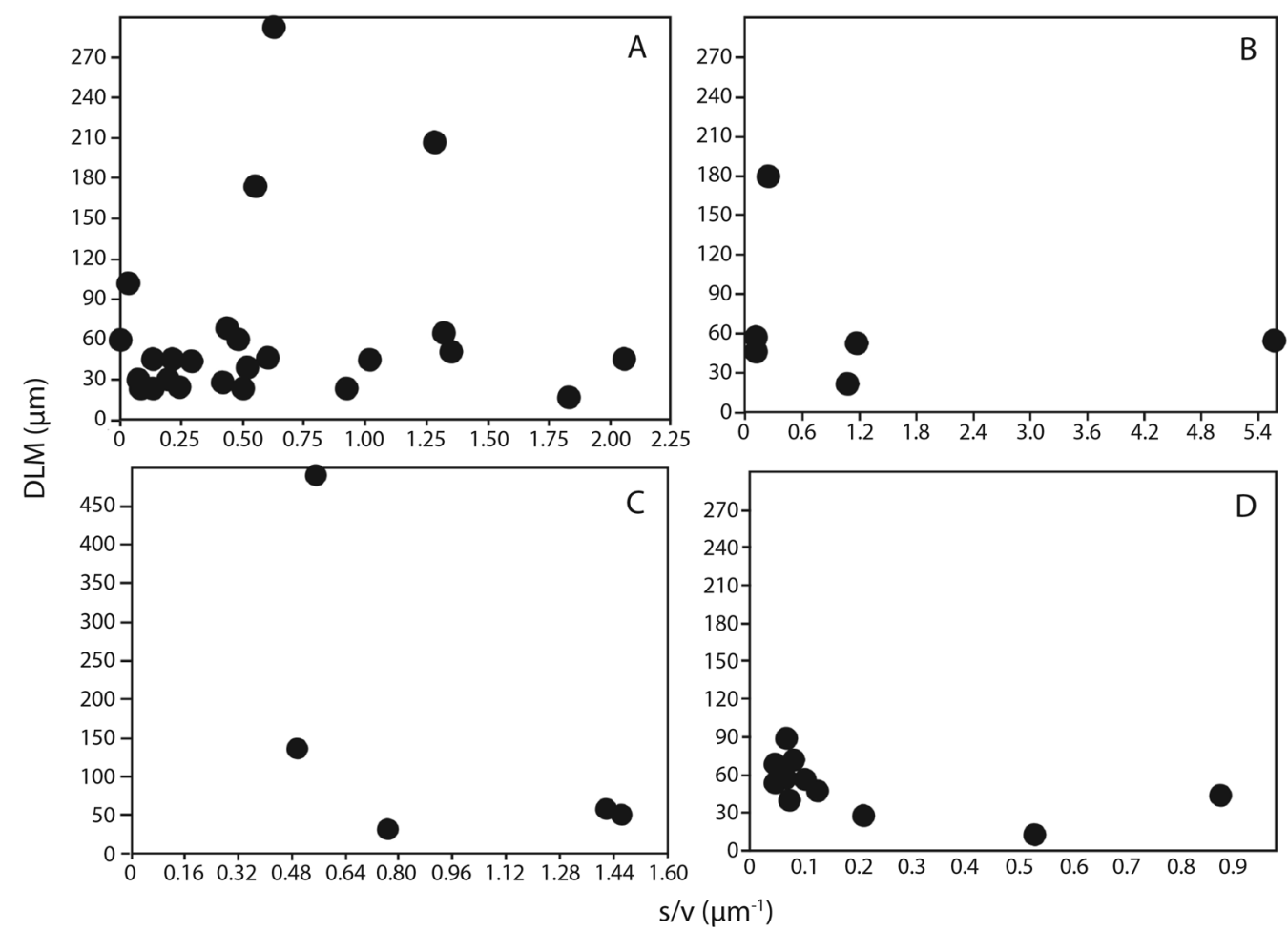

Fig. 3. Relación entre la Dimensión Linear Máxima (DLM) y el cociente Superficie/Volumen (S/V) de cuarenta y nueve taxa de fitoplancton del lago de Tota (Boyacá-Colombia), separadas por GFBM: $\mathbf{A}=$ GFBM IV, B= GFBM V, C= GFBM VI y $\mathbf{D}=$ GFBM VII.

Fig. 3. Relationship between the Greatest Linear Dimension and Surface/Volumen ratio for the forty-nine taxa of phytoplankton of Tota Lake (Boyacá-Colombia), separated by MBFG: $\mathbf{A}=$ MBFG IV, $\mathbf{B}=\mathrm{MBFG}$ V, $\mathbf{C}=\mathrm{MBFG} \mathrm{VI}$ and $\mathbf{D}=$ MBFG VII.

GFBM IV. Para el lago Grande también se evidenció este patrón, que se repite para los meses de febrero y marzo. En menor proporción, se encontraron los GFBM V y VI, cuyo aporte de biovolumen fue menor.

Respecto al ACC, este explicó el 72.8\% de la varianza en los dos primeros ejes canónicos (Fig. 5). Mostró una relación significativa entre el biovolumen agrupado en categorías de forma y las variables ambientales (Test de Monte-Carlo, p<0.05). El eje uno $(49.9 \%)$ presentó correlación positiva con el porcentaje de saturación $(r=0.864)$, el oxígeno disuelto $(\mathrm{r}=0.827)$, la temperatura $(r=0.657)$ y negativa con el nitrógeno total
Kjeldahl ( $\mathrm{r}=-0.597)$. El segundo (22.9\%), se asoció positivamente con la dureza $(\mathrm{r}=0.361)$ y negativamente con la transparencia Secchi $(\mathrm{r}=0.5835)$. Además, se pudo establecer que el biovolumen acumulado por la forma doble cono (E) se relacionó con valores altos de dureza, durante los meses de cota de nivel alta (octubre y noviembre). Las categorías B1 y B2 se asociaron con los valores más bajos de transparencia Secchi, mientras que las algas de la categoría A (esfera) con baja conductividad eléctrica.

Asimismo, el biovolumen acumulado por los organismos asociados a la forma esfera prolongada $(\mathrm{C})$, se asoció con valores de NTK bajos. En tanto, las categorías F, E1, 


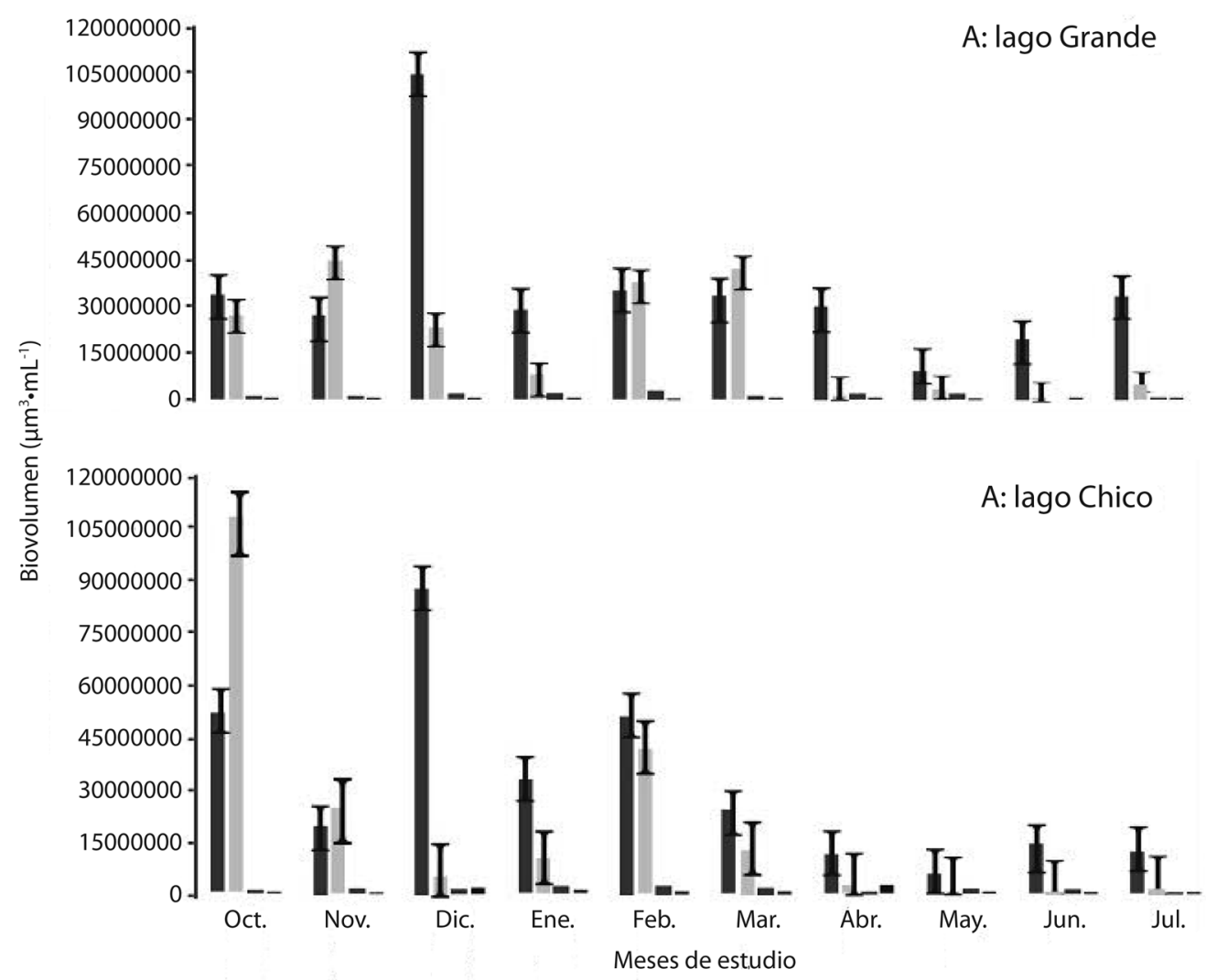

Fig. 4. Distribución temporal del biovolumen por Grupo Funcional Basado en Morfología (GFBM), en los sectores del lago del Tota. A= Lago Grande, B=Lago Chico. Las barras corresponden a los GFBM en orden de importancia (IV-VII-V-VI).

Fig. 4. Temporal Biovolume distribution by Morphologically Based Functional Group (MBFG), in Tota Lake's sectors. A= Lake Grande, B=Lake Chico. Bars correspond to MBFG in order of importance (IV-VII-V-VI).

$\mathrm{H}$ y $\mathrm{D}$, se relacionaron con valores altos de fósforo total, principalmente en los meses donde el nivel del lago fue bajo.

\section{DISCUSIÓN}

El alto grado de aislamiento, la tendencia a la polimixis y el predominio de una condición oligotrófica, son características de los lagos de alta montaña tropical (Löffler, 1962; Lewis, 1996; Catalán \& Donato-Rondón, 2016). Particularmente, ambientes de origen tectónico-glaciar, como es el caso del Lago de Tota, tienden a ser estables, muy transparentes y con baja concentración de nitrógeno (Wetzel, 2001).
Sin embargo, el ordenamiento de las condiciones limnológicas en este trabajo, se dio principalmente por la variación del porcentaje de saturación de oxígeno, la transparencia medida con el disco Secchi, el NTK, la dureza y la alcalinidad, que se explicaron en función de cambios estacionales en el nivel del lago.

Este patrón indica un cambio respecto de su condición natural de lago de alta montaña, así la reducción histórica y la variabilidad estacional de la transparencia se relacionaría principalmente con el incremento del fitoplancton, asociado a su vez con el enriquecimiento de nutrientes (Vollenweider, 1983; Corpoboyacá, 2016). El distanciamiento de un estado 


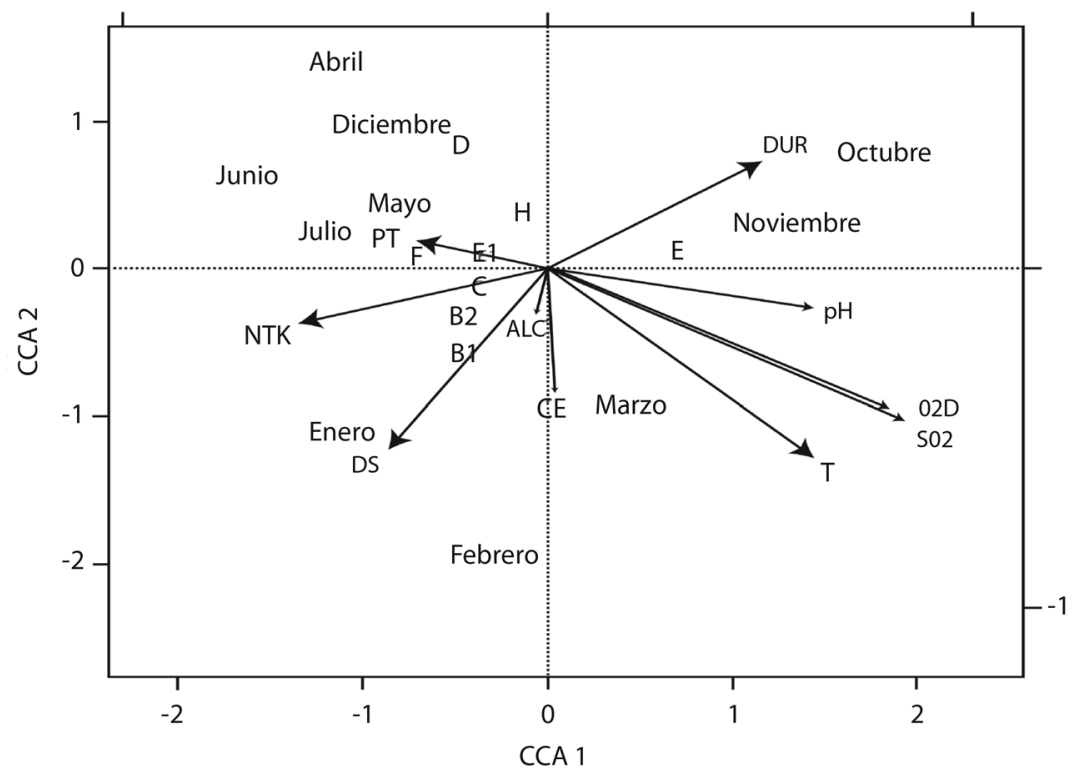

Fig. 5. Análisis de Correspondencia Canónica (ACC) para las variables físicas y químicas y el biovolumen $\left(\mu \mathrm{m}^{3} \cdot \mathrm{mL}^{-1}\right)$ agrupado en categorías de forma (A: esfera; B1: + elipsoide + conos; B2: media esfera; C: esfera prolongada; D: doble cono; E1: doble cono truncado; F: caja rectangular; H: prisma sobre paralelogramo). DS: transparencia Secchi; ALC: alcalinidad; DUR: dureza; C-E: conductividad eléctrica; T: temperatura; O2-D: oxígeno disuelto; SAT O2: saturación de oxígeno; PT: fósforo total; NTK: nitrógeno total Kjeldahl.

Fig. 5. Canonical Correspondence Analysis (CCA) for physical and chemical variables and biovolume $\left(\mu \mathrm{m}^{3}\right.$. $\left.\mathrm{mL}^{-1}\right)$ grouped into form categories (A: sphere; B1: cylinder+ ellipsoid+ cones; B2: half sphere; C; prolate sphere; D: cylinder; E: double cone; E1: truncated double cone; F: rectangular box; H: prism on parallelogram). DS: Secchi transparency; ALC: alkalinity; DUR: hardness; C-E: electrical conductivity; T: temperature; O2D: disolved oxygen; SO2: oxygen saturation; PT: total phosphorus; NTK: total Kjeldahl nitrogen.

oligotrófico a uno de mayor trofía se debe a la intensa actividad antrópica en la cuenca, con implicaciones en la reducción gradual del nivel del lago, la capacidad de almacenamiento y la expansión de la zona litoral (Vollenweider, 1983; Cañón \& Valdés, 2011).

El aumento del contenido de nitrógeno se explica por la descarga directa de afluentes ricos en materia orgánica de origen antrópico, junto con la escorrentía y lavado de las áreas cultivadas, fenómeno más intenso en el sector del lago Chico. La variación estacional de la concentración de nutrientes (PT y NTK) se relaciona con los cambios de nivel del lago, con incremento del NTK en períodos de lluvias y cota de nivel alta por arrastre desde la cuenca, opuesto al comportamiento del PT, que se concentra en los períodos de baja precipitación y cota de nivel bajo. Las cantidades de NTK y PT pueden ser incrementadas, especialmente en el sector del Lago Chico, por la entrada de aguas residuales de la población de Aquitania (Vollenweider, 1983; Cordero et al., 2005; Corpoboyacá, 2016).

Con respecto al fitoplancton, la mayoría de taxa encontrados en el estudio presentaron baja relación $\mathrm{S} / \mathrm{V}\left(<1 \mu \mathrm{m}^{-1}\right)$. Según Margalef (1978) y Reynolds (1988), este atributo puede traducirse en tasas bajas de renovación para los organismos, en respuesta a un bajo estrés por limitación de recursos. Para el Lago de Tota, este rasgo se relaciona principalmente con el enriquecimiento de fósforo (Hernández-Atilano et al., 2012). Aunque menos representativos, los taxa de mayor relación $\mathrm{S} / \mathrm{V}\left(>1 \mu \mathrm{m}^{-1}\right)$ correspondieron a formas 
distorsionadas de la esfera (Lewis, 1976) como por ejemplo Cryptomonas cf. ovata y Gymnodinium sp. Estos dos patrones generales también se encontraron en otros ambientes de la zona andina colombiana. Modesto-Iregui, Guillot, Donato-Rondón y Ortegón (2002), mediante el cálculo de dimensiones fractales de las algas de las lagunas de Fúquene (2540 msnm) y de San Rafael (3300 msnm), repor$\tan$ valores de este cociente que varían entre 0 y $3 \mu \mathrm{m}^{-1}$, que asocian con el estado trófico de los sistemas estudiados, que igualmente se distancia de la oligotrofía por afectación de carácter antrópico y que propicia el desarrollo de especies que amplían su área superficial como mecanismo de captación lumínica, por la eventual disminución de la zona fótica, y desarrollan ultraestructuras celulares complejas que regulan la entrada de los nutrientes.

Además, se identificaron cuatro Grupos Funcionales Basados en Morfología: IV, V, VI y VII, de los cuales los GFBM IV y VII fueron los más representativos. Según Kruk et al. (2010), el primero se caracteriza por sus pérdidas moderadas por sedimentación y tolerancia moderada a la limitación de recursos; el segundo, por agrupar algas con capa mucilaginosa, rasgo que aumenta su volumen y que se traduce en baja relación $\mathrm{S} / \mathrm{V}$. Haciendo un análisis por cada sector del sistema estudiado, son grupos constantes a escala temporal. Aunque hay variaciones sutiles, como ocurre en los meses de octubre y noviembre, donde la cota de nivel presenta sus valores máximos (3014.88 msnm), el grupo VII se acentuó más, sobretodo en el lago Chico. Al reducirse la cota, se evidenció un recambio por el GFBM IV. Para el lago Grande, también se evidenció este patrón, que se repite para los meses de febrero y marzo. Hernández-Atilano et al. (2011) para el mismo sistema sugieren que la dominancia de estos GFBM se debe a que sus características morfológicas, previamente descritas, les permiten controlar su posición en la columna de agua, lo que es concordante con su constancia durante el estudio.

También se registraron los GFBM V y VI. El primero, agrupa algas de alta relación superficie/volumen y provistas de flagelos, características que les permiten enfrentar el hundimiento (Kruk et al., 2010). El segundo, incluye a las diatomeas cuyo rasgo de mayor importancia es la presencia de pared de sílice, la cual les concede ventaja en cuanto al pastoreo, sumada a la formación de colonias y filamentos, que son un importante rasgo para la captación lumínica (Padisák, Soróczki-Pintér, \& Rezner, 2003; Kruk et al., 2010). Aunque estos grupos son menos representativos, en el sector Lago Chico dado su nivel de tensión, el aporte de biovolumen de estos es un poco más elevado que en el Lago Grande. Este patrón difiere de la dominancia de diatomeas y algas euglenófitas reportada para la laguna San Rafael por Modesto-Iregui et al. (2002), que aunque no implementaron la metodología de clasificación del fitoplancton en grupos funcionales, puede traducirse en relevancia de los grupos en mención.

Esta diversidad a nivel funcional puede ser explicada además, para el lago de Tota, por su carácter polimíctico y la fluctuación, a corto plazo, del suministro nutritivo. Constantes movimientos en la columna de agua, se traducen en variación en el entorno físico y químico, proceso que estimula a su vez, el recambio o diversificación del fitoplancton (Weithoff, Walz, \& Gaedke, 2001; Weithoff, 2003).

Es importante mencionar que se encontraron valores de biovolumen que oscilaron entre $1.6 \times 10^{4}$ y $1.05 \times 10^{8} \mu \mathrm{m}^{3} \cdot \mathrm{mL}^{-1}$ y que son mayores a los reportados por HernándezAtilano et al. (2012) para este lago, correspondientes a muestreos realizados entre 2005 y 2007. La toma de muestras del presente trabajo se efectuó entre 2013 y 2014, lo que indica que en solo seis años ya se vislumbran cambios importantes en el sistema y en la respuesta de sus comunidades, en este caso, el fitoplancton.

Por otra parte, la respuesta del fitoplancton del lago de Tota se explicó con base en la relación significativa encontrada entre el biovolumen acumulado según categorías de forma y las variables ambientales. Este fenómeno brinda una aproximación al entendimiento del funcionamiento del sistema acuático 
(Brasil \& Huszar, 2011), ya que proporciona o condiciona formas biológicas según la oferta nutricional y condición limnológica del cuerpo de agua (Modesto-Iregui et al., 2002). La forma doble cono (E), que agrupa especies como Closterium acutum, Elakatothrix cf. gelatinosa y Monoraphidium cf. minutum, se relacionó con la alta dureza, que en el estudio se evaluó en forma de carbonato de calcio. Según Reynolds (2006), estos iones son importantes para el fitoplancton, pues constituyen fuentes adicionales de carbono para realizar la fotosíntesis y generar nueva biomasa. Esta afirmación, concuerda para el lago de Tota, ya que la mayoría de los representantes de la categoría mencionada, presentaron los biovolúmenes más representativos del estudio, principalmente, donde el nivel fue alto y se dio mayor arrastre de materiales de la cuenca.

Condiciones de baja transparencia Secchi en el lago de Tota, se ajustaron mejor a las categorías B1 y B2, cuyos representantes fueron especies como Ceratium hirundinella y Staurodesmus connatus, respectivamente, las cuales tienen como característica común que sus células presentan proyecciones similares a espinas y se asocian a ambientes influenciados por efecto de mezcla (Reynolds et al., 2002). Las modificaciones estructurales son un mecanismo que facilita el incremento del área de captación lumínica, sin ser costoso en términos energéticos y que revela aspectos de la autoecología de las especies que les permiten mantenerse en dadas condiciones de su entorno (Margalef, 1983; Modesto-Iregui et al., 2002; Padisák et al., 2003). Esto sugiere, que la luz es un factor altamente determinante en los ajustes morfológicos del fitoplancton del cuerpo de agua estudiado, que presenta drásticas variaciones en la transparencia y por ende, en la oferta de energía radiante.

La categoría A (esfera), que en su mayoría agrupa algas mucilaginosas como Asterococcus sp. y Sphaerocystis sp., se relacionó con baja conductividad eléctrica. Según Talling (2009), esta variable se aproxima a una medida de la concentración de sustancias inorgánicas y se relaciona con la naturaleza geoquímica de la cuenca. Por su origen tectónico con influencia glaciar, el contenido iónico del lago de Tota es limitado, característica que explicaría la relación con algas que desarrollan mucilago. Este rasgo morfológico les permite aumentar la superficie de almacenamiento de sustancias, principalmente cuando se encuentran restringidas en el medio (Reynolds, 2006).

La categoría C (esfera prolongada) se asoció a condición de bajo NTK. Las algas aquí agrupadas, por ejemplo Oocystis cf. parva o Scenedesmus ecornis, carecen de rasgos morfológicos particulares como mucilago o espinas. Por esta razón, su respuesta a la limitación de nitrógeno, se basa en la deformación de alguna de sus dimensiones celulares para reducir su relación superficie/volumen (Reynolds, 1997; Modesto-Iregui et al., 2002). Este rasgo optimiza la captación de este nutriente, que como se menciona, presenta una variación estacional.

Condiciones de alta concentración de fósforo total, principalmente en meses donde el nivel del lago fue bajo, se ajustaron mejor a las categorías caja rectangular $(\mathrm{F})$, prisma sobre paralelogramo $(\mathrm{H})$, doble cono truncado (E1), y (D), las cuales agrupan especies como Asterionella formosa, Nitzchia sp., Staurastrum chaetoceras y Aulacoseira granulata, que son reportadas como asociaciones características de lagos eutróficos (Duque \& Donato-Rondón, 1992; Reynolds et al., 2002). La presencia de esta asociación de algas, es reflejo de la tendencia que tiene el lago de aumentar su estado trófico, según la afirmación de Cardozo et al. (2005), característica no esperada para un ambiente de alta montaña tropical y del efecto que tienen las variaciones de su nivel en la dinámica de los nutrientes. Con respecto a la morfología, estas especies tienen grandes valores de DLM, rasgo que se relaciona con condiciones cambiantes de estabilidad en ambientes de alta categoría trófica (Naselli-Flores \& Barone, 2003).

El presente estudio ha documentado que la respuesta morfológica y funcional del fitoplancton del lago de Tota está condicionada principalmente por la variabilidad a corto plazo, en las condiciones de transparencia, fósforo, 
nitrógeno, dureza y conductividad eléctrica, que son influenciadas por los cambios estacionales en la cota de nivel, reflejan asociaciones que van más allá del nivel taxonómico y develan gran diversidad ecológica en este ambiente (Reynolds, 1988). Es destacable el hecho de que las categorías de forma agrupen algas de diferentes grupos funcionales, lo que indica la necesidad de hacer ajustes a los sistemas de clasificación funcional, vinculando cada aspecto particular que presentan los ambientes ubicados en la provincia de alta montaña tropical.

\section{AGRADECIMIENTOS}

A la Universidad Pedagógica y Tecnológica de Colombia por la financiación del proyecto a través de la convocatoria DIN-capital semilla 2013. A Carla Kruk por sus valiosos aportes y sugerencias, a Alejandra Jiménez y Nidia Gil por el acompañamiento en la fase campo, a Ángela Zapata y Carlos Rivera por su aporte en la labor taxonómica y a las integrantes del grupo de investigación de Jonathan Shurin, de la Universidad de California-San Diego, por su revisión del abstract y las demás secciones escritas en inglés.

\section{RESUMEN}

La morfología funcional corresponde a las respuestas de adaptación a los cambios en el medio ambiente. En fitoplancton, rasgos como el desarrollo de mucílago, vesículas de aceite, filamentos y variación en la relación superficie/ volumen, permiten enfrentar la sedimentación, optimizar la captación lumínica y de nutrientes. El objetivo del presente estudio fue evaluar la relación entre los rasgos y la variación de las condiciones físicas y químicas en el lago de Tota (Boyacá, Colombia). Para esto, se realizaron diez muestreos mensuales entre octubre 2013 y julio 2014. Se midieron el $\mathrm{pH}$, conductividad eléctrica, temperatura, oxígeno disuelto, saturación de oxígeno, alcalinidad y dureza total del agua. Además se recolectaron muestras para la cuantificación en laboratorio de Nitrógeno Total Kjeldahl y Fósforo Total. Las algas se obtuvieron en diferentes profundidades según la transparencia Secchi. Se caracterizó la composición taxonómica y se estimó la abundancia por el método de sedimentación en cámaras. Se midieron los rasgos morfológicos de cada taxa según la correspondencia a modelos geométricos, se clasificaron en grupos funcionales basados en morfología (GFBM) y se calculó su biomasa expresada como biovolumen. Las variables fueron evaluadas mediante Análisis de Componentes Principales, que incluyó el efecto espacio-temporal y fue interpretado según la variación en el nivel del agua. Mediante Análisis de Correspondencia Canónica se estableció la relación entre el biovolumen del fitoplancton acumulado en categorías de forma y las variables ambientales. La ordenación mostró que las condiciones limnológicas son influenciadas por cambios estacionales, principalmente representadas por el porcentaje de saturación de oxígeno, la transparencia Secchi, el NTK, la dureza y la alcalinidad. Se encontraron cuatro GFBM: IV, V, VI y VII, siendo los GFBM IV y VII, los más representativos. El ACC fue significativo (Test de Monte-Carlo, $\mathrm{p}<0.05$ ). En conclusión, la respuesta morfológica y funcional del fitoplancton del lago de Tota esta condicionada principalmente por la variabilidad a corto plazo, en la transparencia, el fósforo, el nitrógeno, la dureza y la conductividad eléctrica, que son afectados por los cambios estacionales en el nivel del lago.

Palabras clave: biovolumen, fitoplancton, grupo funcional, lago de Tota, morfología funcional.

\section{REFERENCIAS}

American Public Health Association (APHA), American Water Works Association (AWWA), \& Water Environment Federation (WEF). (2012). Standard Methods for examination of water and wastewater (22nd edition). Washington, D.C.: APHA.

Armada Nacional. (1998). Levantamiento topográfico, batimétrico y sedimentológico laguna de Tota. Cartagena de Indias, Colombia (Informe final). Colombia: Centro de Investigaciones Oceanográficas e Hidrológicas.

Brasil, J., \& Huszar, V. L. M. (2011). O papel dos traços funcionais na ecologia do fitoplâncton continental. Oecologia Australis, 15(4), 799-834. doi: 10.4257/ oeco.2011.1504.04

Cañón, J., \& Valdés, J. (2011). Assessing the Influence of Global Climate and Anthropogenic Activities on the Water Balance of an Andean Lake. Journal of Water Resource and Protection, 3, 883-891.doi: 10.4236/ jwarp.2011.312098

Cardozo, A., Bermúdez, A., Aranguren-Riaño, N., \& Duque, S. R. (2005). Algas planctónicas del lago de Tota: listado taxonómico actualizado. Ciencia en desarrollo, 2(1), 80-88.

Castellanos, L., \& Donato-Rondón, J. (2008). Biovolumen y sucesión de diatomeas bénticas. In J. DonatoRondón (Ed.), Ecología de un río de montaña de los Andes Colombianos (Rio Tota - Boyacá) (pp. 127144). Bogotá: Centro de Publicaciones Universidad Nacional de Colombia. 
Catalán, J., \& Donato-Rondón, J. (2016). Perspectives for an integrated understanding of tropical and temperate high-mountain lakes. Journal of Limnology, 75(s1), 215-234. doi: 10.4081/jlimnol.2016.1372

Cordero, R., Ruiz, E., \& Vargas, E. (2005). Determinación espacio-temporal de la concentración de fósforo en el lago de Tota. Revista Colombiana de Química, 34(2), 211-218.

Corpoboyacá, (2016). Efecto de variabilidad climática de un ciclo anual sobre el flujo de nutrientes $(C, N y P)$, fuentes y biocaptación en el Lago de Tota (Informe técnico). Tunja, Colombia: Centro de documentación de la Corporación Autónoma Regional de Boyacá.

Duque, S. \& Donato-Rondón, J. (1992). Biología y Ecología del fitoplancton de las aguas dulces en Colombia. Cuadernos Divulgativos, 35, 1-21.

Esteves, F. A., \& Suzuki, M. S. (2011). Comunidade Fitoplanctônica. In F. A. Esteves (Ed.), Fundamentos de Limnología $3^{\circ}$ edición (pp. 375-445). Rio de Janeiro: Editora Interciência.

García-Berthou, E., Alcaraz, L., Benejam, L., \& Benito, J. (2009). Diseño experimental y análisis de datos. In A. Elosegi \& S. Sabater (Eds.), Conceptos y técnicas en ecología fluvial (pp. 397-412). Bilbao: Editorial Fundación BBVA.

Gómez, M., Rivera, C., Duque, S., Andrade, C., \& Rodríguez, C. (2011). Análisis temporal y espacial del fitoplancton en el sistema de lagos Yahuarca, Planicie de Inundación del Río Amazonas (Leticia, Colombia). El Astrolabio, Revista de Investigación y Ciencia del Gimnasio Campestre, 1, 7-27.

Graham, L., Graham, J., \& Wilcox, L. (2009). Algae $\left(2^{\text {nd }}\right.$ edition $)$. San Francisco: Pearson Benjamin Cummings.

Grime, J. (1977). Evidence for the existence of three primary strategies in plants and its relevance to ecological and Evolutionary Theory. The American Naturalist, 111(982), 1169-1194.

Harris, G. (1986). Phytoplankton Ecology: Structure, Function and Fluctuation. New York: Chapman \& Hall.

Hernández-Atilano, E., Aguirre-Ramírez, N., RamírezRestrepo, J., Duque, S. R., Guisande-González, C., Bonilla, S., \& Kruk, C. (2011). Grupos funcionales basados en la morfología del fitoplancton en lagos tropicales. Boletín de la Sociedad Argentina de Botánica, 46(sl 2), 83-84.

Hernández-Atilano, E., Aguirre-Ramírez, N., Palacio-Baena, J., Ramírez-Restrepo, J., Duque, S., Guisande-González, C., Aranguren-Riaño, N., \& Mogollón, M. (2012). Rasgos morfológicos del fitoplancton en seis sistemas leníticos de las regiones Amazónica, Andina y Caribe de Colombia. Actualidades Biológicas, 34(96), 67-83.

Hernández-Atilano, E., Aguirre-Ramírez, N., Palacio-Baena, J., Ramírez-Restrepo, J., Duque, S., GuisandeGonzález, C., Aranguren-Riaño, N., \& Mogollón, M. (2013). Evaluación comparativa de algunas características limnológicas de seis ambientes leníticos de Colombia. Revista Facultad de Ingenieria Universidad de Antioquia, 69, 216-228.

Hillebrand, H., Dürselen, C., Kirschtel, D., Pollingher, U., \& Zohary, T. (1999). Biovolume calculations for pelagic and benthic microalgae. Journal of Phycology, 35(2), 103-421.

Huszar, V. L. M., \& Reynolds, C. S. (1997). Phytoplankton periodicity and sequences of dominance in an Amazonian floodplain lake (Lago Batata, Pará, Brazil): Responses to gradual environmental change. Hydrobiologia, 346, 169-181. doi: 10.1023/A:1002926318409

Huszar, V. L. M., Silva, L. H. S., Marinho, M., Domingos, P., \& Sant'Anna, C. L. (2000). Cyanoprokaryote assemblages in eight productive tropical Brazilian waters. Hydrobiologia, 424, 67-77. doi: 10.1023/A: 1003996710416

Hutchinson, G. E. (1957). Concluding remarks. Cold spring Harbor Symposia on Quantitative. Biology, 22, 415-427.

Kruk, C., Huszar, V. L. M, Peeters, E., Bonilla, S., Costa, L., Rling, M., Reynolds. C. S., \& Scheffer, M. (2010). A morphological classification capturing functional variation in phytoplankton. Freshwater Biology, 55, 614-627. doi: 10.1111/j.1365-2427.2009.02298x

Lewis, W. M. (1976). Surface/ Volume Ratio: Implications for Phytoplankton Morphology. Science, 192, 885-887.

Lewis, W. M. (1996). Tropical lakes: How latitude makes a difference. In F. Schiemer, \& K. T. Boland (Eds.), Perspectives in Tropical Limnology (pp. 43-64). Amsterdam: SPB Academic Publishing.

Löffler, H. (1962). The limnology of tropical high-mountain lakes. Internationale Vereinigung fuer Theoretische und Angewandte Limnologie Verhandlungen, 15, 176-193.

Margalef, R. (1978). Life-forms of phytoplankton as survival alternatives in an unstable environment. Oceanica Acta, 1(4), 493-509.

Margalef, R. (1983). Limnología. Barcelona: Ediciones Omega, S. A.

Margalef, R. (1998). Ecología (9a edición). Barcelona: Ediciones Omega, S. A. 
McCune, B., \& Grace, J. (2002). Analysis of Ecological Communities. Gleneden Beach, OR: MjM software design.

Melo, S., \& Huszar, V. L. M. (2000). Phytoplankton in an Amazonian flood-plain lake (Lago Batata, Brasil): diel variation and species strategies. Journal of Plankton Research, 22, 63-76. doi: 10.1093/ plankt/22.1.63

Modesto-Iregui, M., Guillot, G., Donato-Rondón, J., \& Ortegón, M. (2002). Dimensión fractal y relación área superficial/volumen de algas del fitoplancton de lagos colombianos. Caldasia, 24(1), 121-134.

Naselli-Flores, L., \& Barone, R. (2003). Steady-state assemblages in a mediterranean hypertrophic reservoir. The role of Microcystis ecomorphological variability in maintaining an apparent equilibrium. Hydrobiologia, 502, 133-143.

Noriega, J., Castillo, D., Vásquez, A., \& Monroy, D. (2010). Estudio preliminar del estado de la microcuenca Los Pozos y su posible efecto en un sector de la laguna de Tota, Boyacá-Colombia. Revista de Tecnología, 9(2), 145-164.

Padisák, J., Crossetti, L. O., \& Naselli-Flores, L. (2009). Use and misuse in the application of the phytoplankton functional classification: a critical review with updates. Hydrobiologia, 621, 1-19. doi: 10.1007/ s10750-008-9645-0

Padisák, J., Soróczki-Pintér, E., \& Rezner, Z. (2003). Sinking properties of some phytoplankton shapes and the relation of form resistance to morphological diversity of plankton - an experimental study. Hydrobiologia, $500,243-257$.

Ramírez-Restrepo, J. (2000). Fitoplancton de agua dulce, aspectos ecológicos, taxonómicos y sanitarios. Medellín: Editorial Universidad de Antioquia.

Rangel O., \& Aguirre, J. (1983). Comunidades acuáticas altoandinas - I. Vegetación sumergida y de ribera en el lago de Tota, Boyacá, Colombia. Caldasia, $65,719-742$.

Reynolds, C. S. (1980). Phytoplankton assemblages and their periodicity in stratifying lake systems. Holarctic Ecology, 3, 141-159.

Reynolds, C. S. (1988). Functional Morphology and adaptive strategies of freshwater phytoplankton. In C. D. Sandgren (Ed.), Growth and reproductive strategies of freshwater phytoplankton (pp. 388-433). Cambridge: Cambridge University Press.

Reynolds, C. S. (1997). Vegetation processes in the pelagic: a model for ecosystem theory. Germany: Ecology Institute.
Reynolds, C. S. (2006). The ecology of phytoplankton. Nueva York: Cambridge University Press.

Reynolds, C. S., Huszar, V. L. M., Kruk, C., NaselliFlores, L., \& Melo, S. (2002). Towards a functional classification of the freshwater phytoplankton. Journal of Plankton Research, 24(5), 417-428. doi: 10.1093/plankt/24.5.417

Rhee, G. (1982). Effects of environmental factors and their interactions on the phytoplankton growth. Advances in Microbial Ecology, 6, 33-73. doi: 10.1007/978-1-4615-8318-9 2

Roldán-Pérez, G. \& Ramírez-Restrepo, J. (2008). Fundamentos de Limnología neotropical ( $2^{\mathrm{a}}$ edición). Medellín: Editorial Universidad de Antioquia.

Ros, J. (1979). Prácticas de Ecología. Barcelona: Editorial Omega Barna.

Rott, E. (1981). Some results from phytoplankton counting intercalibrations. Schweizerische Zeitschrift fur Hydrologie-swiss Journal of Hydrology, 43, 34-62. doi: 10.1007/BF02502471

Ruiz, E. (2002). Métodos para el estudio de las características Físico-químicas del agua. In G. Rueda (Ed.), Manual de Métodos en Limnología (pp. 9-27). Bogotá: Asociación Colombiana de Limnología (ASCOLIMNOS).

Salmaso, N., \& Padisák, J. (2007). Morpho-Functional Groups and phytoplankton development in two deep lakes (Lake Garda, Italy and Lake Stechlin, Germany). Hidrobiologia, 578, 97-112. doi: 10.1007/ s10750-006-0437-0

Schwoerbel, J. (1975). Métodos de hidrobiología. Madrid: Ediciones Blume.

Smith, V. H., \& Schindler, D. W. (2009). Eutrophication science: where do we go from here? Trends in Ecology and Evolution, 24, 201-207.

Souza, M. B., Barros, C. F., Barbosa, F., Hajnal, E., \& Padisák, J. (2008). Role of atelomixis in replacement of phytoplankton assemblages in Dom Helvécio Lake, South-East Brazil. Hydrobiologia, 607, 211-224.

Sun, J., \& Liu, D. (2003). Geometric models for calculating cell biovolume and surface area for phytoplankton. Journal of Plankton Research, 25(11), 1331-1346. doi: 10.1093/plankt/fbg096

Talling, J. F. (2009). Electrical conductance - a versatile guide in freshwater science. Freshwater Reviews, 2, 65-78. doi: 10.1608/FRJ-2.1.4

Ter Braak, C. J. (1986). Canonical Correspondence Analysis: A New Eigenvector Technique for Multivariate 
Direct Gradient Analysis. Ecology, 67(5), 1167-1179. doi: $10.2307 / 1938672$

Utermöhl, H. (1958). Zur Vervollkommnung der quantitative Phytoplankton-Methodik. Mitteilungen des Internationalen Limnologie, 9, 1-38.

Vollenweider, R. (1983). Informe sobre el Lago de Tota. Bogotá, Colombia (Informe técnico). Colombia, Corporación Autónoma Regional de Cundinamarca.

Weithoff, G. (2003). The concepts of 'plant functional types' and 'functional diversity' in lake phytoplankton - a new understanding of phytoplankton ecology? Freshwater Biology, 48, 1669-1675. doi: 10.1046/j.1365-2427.2003.01116.x

Weithoff, G., Walz, N., \& Gaedke, U. (2001). The intermediate disturbance hypothesis-species diversity or functional diversity? Journal of Plankton Research, 23(10), 1147-1155. doi: 10.1093/plankt/23.10.1147

Wetzel, R. (2001). Limnology: Lake and River Ecosystems ( $3^{\text {rd }}$ edition). San Diego, California Academic Press.

Wetzel, R., \& Likens, G. (2000). Limnological Analyses ( $2^{\text {nd }}$ edition). New York: Springer Science + Business Media. 
